\title{
Radioactive Ion Beam studies of $\alpha, p$ process waiting points in X-Ray Bursts
}

\section{M. Deibel ${ }^{* \dagger}$}

Department of Physics and Astronomy, Louisiana State University, Baton Rouge, LA 70803 USA Joint Institute for Nuclear Astrophysics, Michigan State University, East Lansing, MI 48824 USA Physics Division, Argonne National Laboratory, Argonne, IL 60439 USA

E-mail: deibelephys.1su.edu

\section{Afanasieva, J. Lai, B. C. Rasco}

Department of Physics and Astronomy, Louisiana State University, Baton Rouge, LA 70803 USA

M. Albers, M. Alcorta, S. Almarez-Calderon, P. F. Bertone, J. Chen, J. A. Clark, J. P. Greene, C. R. Hoffman, C. L. Jiang, B. P. Kay, H. Y. Lee, C. Nair, T. Palachan-Hazan, R. C. Pardo, K. E. Rehm, A. M. Rogers, C. Ugalde, G. Zinkann

Physics Division, Argonne National Laboratory, Argonne, IL 60439 USA

\section{S. Bedoor, D. Shetty, A. Wuosmaa}

Physics Department Western Michigan University, Kalamazoo, MI 49008 USA

\section{P. Carnelli, J. M. Figueira}

Laboratorio Tandar, Comisión Nacional de Energía Atómica, B1650KNA San Martín, Buenos Aires, Argentina

\author{
A. A. Chen, D. Irvine, S. Manwell \\ Department of Physics and Astronomy, McMaster University, Hamilton, ON L8S 4M1, Canada
}

\section{J. C. Lighthall, S. T. Marley}

Physics Division, Argonne National Laboratory, Argonne, IL 60439 USA

Physics Department Western Michigan University, Kalamazoo, MI 49008 USA

\section{N. Patel}

Physics Division, Argonne National Laboratory, Argonne, IL 60439 USA

Department of Physics, Colorado School of Mines, Golden, CO 80401 USA

M. Paul 


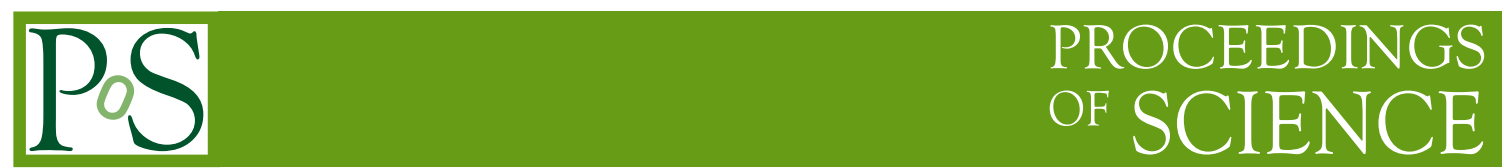

Racah Institute of Physics, Hebrew University, Jerusalem, Israel

The nucleosynthetic flow in type I X-ray Bursts (XRBs) is driven by the triple- $\alpha, r p$ and $\alpha, p$ processes. Several intermediate mass nuclei, ${ }^{22} \mathrm{Mg},{ }^{26} \mathrm{Si},{ }^{30} \mathrm{~S}$, and ${ }^{34} \mathrm{Ar}$, have been identified as possible candidates for waiting points in XRBs. When such a nucleus is reached, the flow stalls due to a $(p, \gamma)-(\gamma, p)$ equilibrium and must await $\beta$ decay unless the $(\alpha, p)$ reaction is fast enough to break out of the waiting point first. A method to study these $\alpha p$-process reactions has been developed whereby the time-inverse reaction is studied in inverse kinematics using radioactive ion beams produced by the in-flight method at the Argonne National Laboratory ATLAS facility. These time-inverse reactions have been used to study all four of the $\alpha, p$ process waiting points via the $p\left({ }^{25} \mathrm{Al},{ }^{22} \mathrm{Mg}\right) \alpha, p\left({ }^{29} \mathrm{P},{ }^{26} \mathrm{Si}\right) \alpha, p\left({ }^{33} \mathrm{Cl},{ }^{30} \mathrm{~S}\right) \alpha$, and $p\left({ }^{37} \mathrm{~K},{ }^{34} \mathrm{Ar}\right) \alpha$ reactions. The data from these studies have been used to determine rates for ${ }^{22} \mathrm{Mg}(\alpha, p){ }^{25} \mathrm{Al},{ }^{26} \mathrm{Si}(\alpha, p){ }^{29} \mathrm{P},{ }^{30} \mathrm{~S}(\alpha, p){ }^{33} \mathrm{Cl}$, and ${ }^{34} \operatorname{Ar}(\alpha, p){ }^{37} \mathrm{~K}$ and have also been compared with theoretical calculations. The results and possible implications for nucleosynthesis in XRBs will be discussed.

XII International Symposium on Nuclei in the Cosmos

August 5-12, 2012

Cairns, Australia

* Speaker.

${ }^{\dagger}$ A footnote may follow. 


\section{Introduction}

Type I X-ray bursts (XRBs) occur in binary star systems in which hydrogen-rich matter is accreted from a companion, main-sequence star onto the surface of a neutron star at a rate of approximately $10^{-8}-10^{-10} M_{\odot} / y r$ (for an in depth review of X-ray binaries see e.g. [1]). During this accretion phase, while temperatures are approximately $0.1 \mathrm{GK}$, there is a persistent, thermal $\mathrm{X}$-ray emission. However, as matter is accreted, the pressure and density build up on the neutron star's surface and thermonuclear runaway occurs. Temperatures of $1-2 \mathrm{GK}$ are reached during XRBs, which typically last for approximately $10-100 \mathrm{~s}$ while emitting $10^{39}-10^{40} \mathrm{ergs}$ of energy, increasing the luminosity of the system by at least an order of magnitude. After the burst, the accretion phase of the system resumes, and the burst cycle begins again, with typical XRB recurrence times on the order of hours to days. Due to the high density of the neutron star, little if any synthesized material escapes the system and most of the nuclei created during the burst become part of the neutron star crust and seeds for subsequent bursts. As a result, these systems are evolving, with each burst affecting those after it. In addition to the seed nuclei from previous bursts, the initial composition before each burst is also affected by the hot-CNO cycle, which burns hydrogen into helium in between bursts.

The thermonuclear runaway that occurs during these explosions is triggered by the triple- $\alpha$ reaction and then driven up the proton-rich side of the chart of nuclei via the $\alpha, p$ and $r p$ processes. The $r p$ process is a series of rapid proton captures and $\beta$ decays that reaches up to the SnSbTe region, although the actual end point of the $r p$ process is unclear $[2,3]$. In the lower mass regime of the chart of nuclei $(\mathrm{A}=20-40)$, the $\alpha, p$-process occurs. This process consists of a series of $(\alpha, p)$ and $(p, \gamma)$ reactions, which strongly depend on the temperature of the burst due to the large Coulomb barrier of the $(\alpha, p)$ reactions. During this process the nucleosynthetic flow can be stalled at so-called waiting-point nuclei. At such a nucleus a low $\mathrm{Q}_{p, \gamma}$ value results in a $(p, \gamma)-(\gamma, p)$ equilibrium and the nucleosynthetic flow must await $\beta^{+}$decay. There have been four potential $\alpha, p$-process waiting points identified: the $(\mathrm{Z}-\mathrm{N}) / 2=1$ nuclei ${ }^{22} \mathrm{Mg},{ }^{26} \mathrm{Si},{ }^{30} \mathrm{~S}$, and ${ }^{34} \mathrm{Ar}$ [4]. As these nuclei have half-lives on the order of seconds this delay can be significant on XRB time scales. However, if a competing reaction, such as the $(\alpha, p)$ reaction, is fast enough, breakout from the waiting point occurs and nucleosynthesis continues up the chart of nuclides. Therefore, it is important to understand the different processes occurring at a waiting-point nucleus in a self consistent way.

In addition to the typical XRB luminosity profiles, there have been several observations of double-peaked bursts [5, 6, 7], and at least one observation of a triple-peaked burst [8]. Several explanations of these multi-peaked bursts have been proposed including stalling of the nuclear burning front [9]. Another possible explanation for these double and triple-peaked profiles is that the stalling of the nucleosynthetic flow, due to the presence of the $\alpha, p$-process waiting points may cause a decrease in the energy generated by the burst and thus a dip in the luminosity profile [10].

The importance of $(\alpha, p)$ reactions on these waiting points is reinforced by a recent sensitivity study of reaction rates in XRBs [11]. Despite the thousands of reactions that go into XRB nucleosynthesis, by varying these rates by a factor of 10 up and down, the authors of [11] found that only 28 reactions significantly affected the final elemental abundances produced by XRBs and an even smaller number affected the energy generation in these bursts. Among those reactions 
that showed affects on elemental abundances and/or energy generation were several of the $(\alpha, p)$ reactions discussed above.

Despite the apparent importance of these reactions, there is little experimental data available on them and stellar models use theoretical rates based on Hauser-Feshbach theory, which may not be accurate due to the low level densities of the even-even compound nuclei in the $\mathrm{A}=20$ - 40 mass regime. Previously, studying these reaction rates had not been possible due to the lack of radioactive ion beams. Indeed, many of the important reactions in XRBs are still impossible to reach; however, with the advent of radioactive ion beams (RIBs) a few of those important reactions that are closer to stability can now be accessed, including the $(\alpha, p)$-process waiting-point reactions. In addition to RIB studies there have also been recent developments using stable beams to study the levels of the compound nuclei produced in these reactions [12]

We have studied the $(\alpha, p)$ reactions on all four $\alpha, p$ process waiting points by measuring the time inverse $(p, \alpha)$ reactions in inverse kinematics using RIBs produced at the Argonne Tandem Linac Accelerator System (ATLAS) at Argonne National Laboratory. The experimental details of the new techniques used to study these reactions are outlined below as well as the preliminary results of these measurements and their possible effects on XRB nucleosynthesis.

\section{Experiment}

The $p\left({ }^{25} \mathrm{Al},{ }^{22} \mathrm{Mg}\right) \alpha, p\left({ }^{29} \mathrm{P},{ }^{26} \mathrm{Si}\right) \alpha, p\left({ }^{33} \mathrm{Cl},{ }^{30} \mathrm{~S}\right) \alpha$, and $p\left({ }^{37} \mathrm{~K},{ }^{34} \mathrm{Ar}\right) \alpha$ reactions were measured to study the ${ }^{22} \mathrm{Mg}(\alpha, p){ }^{25} \mathrm{Al},{ }^{26} \mathrm{Si}(\alpha, p){ }^{29} \mathrm{P},{ }^{30} \mathrm{~S}(\alpha, p){ }^{33} \mathrm{Cl}$, and ${ }^{34} \mathrm{Ar}(\alpha, p){ }^{37} \mathrm{~K}$ reactions, respectively. Radioactive ion beams of ${ }^{25} \mathrm{Al},{ }^{29} \mathrm{P},{ }^{33} \mathrm{Cl}$ and ${ }^{37} \mathrm{~K}$ were produced at the ATLAS "in-flight" facility using stable beams of ${ }^{24} \mathrm{Mg},{ }^{28} \mathrm{Si},{ }^{32} \mathrm{~S}$, and ${ }^{36} \mathrm{Ar}$. The primary stable beams were accelerated to energies of approximately $315-325 \mathrm{MeV}$ and impinged upon a $\mathrm{LN}_{2}$-cooled gas target filled with 1.4 atm of $\mathrm{D}_{2}$ gas. Radioactive ion beams of ${ }^{25} \mathrm{Al},{ }^{29} \mathrm{P},{ }^{33} \mathrm{Cl}$, and ${ }^{37} \mathrm{~K}$ with energies of 253, 280, 250, and $275 \mathrm{MeV}$, respectively, were produced via the $(d, n)$ reaction. The radioactive ions of interest were then refocused, rebunched and separated by an analyzing magnet from the stable component of the beam. Typically the low energy tail of the unreacted stable beam is also selected by the analyzing magnet resulting in an unfavorable ratio of approximately 1:1000 radioactive ions/s to stable ions/s. As these two components of the resulting "cocktail" beam are separated in time of flight, a radio-frequency (RF) sweeper is used to eliminate much of the stable beam contamination improving the radioactive to stable beam ratio to better than 1:1. The intensities of the RIBs ranged from $1-5 \times 10^{4}$ ions/s depending on the RIB species.

Changing the energy of the RIB to scan through the energy region of interest for the reaction by changing the energy of the primary, stable beam would be prohibitively time consuming given the difficulty of producing and tuning RIBs. Therefore, in order to change beam energies Au degrader foils of different thicknesses were used. The thicknesses of the Au foils were chosen such that the energy loss of the beam in the target would be approximately the same as the energy loss in the foils (approximately $10 \mathrm{MeV}$ in the case of a $250 \mathrm{MeV}{ }^{33} \mathrm{Cl}$ beam).

Once the RIB has been produced it is delivered to the experimental area where it impinges on a $\mathrm{CH}_{2}$ target of approximately $650 \mu \mathrm{g} / \mathrm{cm}^{2}$. The $\alpha$ particles produced from the $(p, \alpha)$ reactions are detected in an annular double-sided Si detector (DSSD) that is segmented in $\theta_{\text {lab }}$ (see Fig. 1). The DSSD was placed such that lab angles of $6^{\circ}-19^{\circ}$ were covered for the $p\left({ }^{25} \mathrm{Al},{ }^{22} \mathrm{Mg}\right) \alpha$ and 


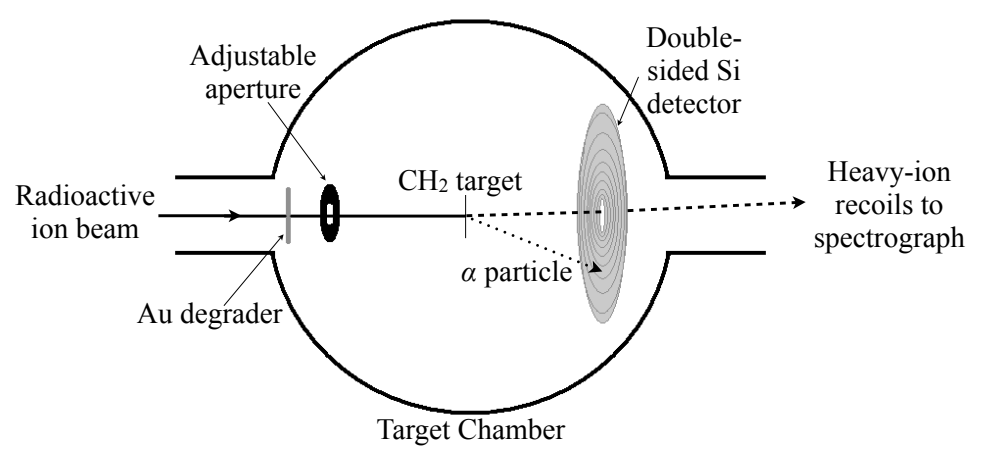

Figure 1: A schematic of the experimental setup.

$p\left({ }^{29} \mathrm{P},{ }^{26} \mathrm{Si}\right) \alpha$ reactions and $8^{\circ}-24^{\circ}$ were covered in the $p\left({ }^{33} \mathrm{Cl},{ }^{30} \mathrm{~S}\right) \alpha$ and $p\left({ }^{37} \mathrm{~K},{ }^{34} \mathrm{Ar}\right) \alpha$ reaction studies.

The heavy-ion recoils along with the unreacted beam (from both the RIB and the stable contaminant) are momentum-analyzed by an Enge split-pole spectrograph. The reaction products of interest are separated from other heavy ions by the spectrograph, which was run in gas-filled mode, filled with approximately 15 Torr of $\mathrm{N}_{2}$ gas in the case of the highest beam energies. The gas-filled mode was used to collapse the charge state distribution of the heavy-ion recoils into a single charge state [13] to maximize detection efficiency at the focal plane, which is crucial in these types of low-statistics experiments, as well as to avoid position overlap of the reaction products of interest and unreacted beam in various charge states. Once the heavy recoils pass through the spectrograph, they are detected at the focal plane by a gas-filled parallel grid avalanche counter (PGAC) and an ionization chamber. These detectors give position and energy loss of the ions, as well as timeof-flight of the ions relative to the RF of the beam, allowing particle identification. By detecting the heavy-ion recoils in coincidence with the $\alpha$ particles, correcting for efficiencies, and normalizing to the incident beam and target thickness, the cross section of the reaction of interest can be determined.

\section{Analysis and Results}

The $\alpha$ particles were detected by the DSSD in coincidence with the heavy-ion recoils as discussed above to determine the $\alpha$-particle yield for the reaction. Background was partially eliminated through particle identification cuts on the time-of-flight and focal plane position (or equivalently magnetic rigidity) of the heavy recoils. Additional background was subtracted by determining the random background rate outside of the coincidence timing cut. Once the $\alpha$-particle yield was determined it was corrected for various efficiencies and normalized to the incident beam and target areal density.

Unfortunately, as the magnetic rigidity of the high-energy heavy recoils associated with the low-energy $\alpha$ branch is the same as the magnetic rigidity of the unreacted beam, those recoils were blocked from entering the detector to avoid overwhelmingly high count rates and only the high-energy $\alpha$ branch of the reaction could be measured. The efficiency factor for detecting only 


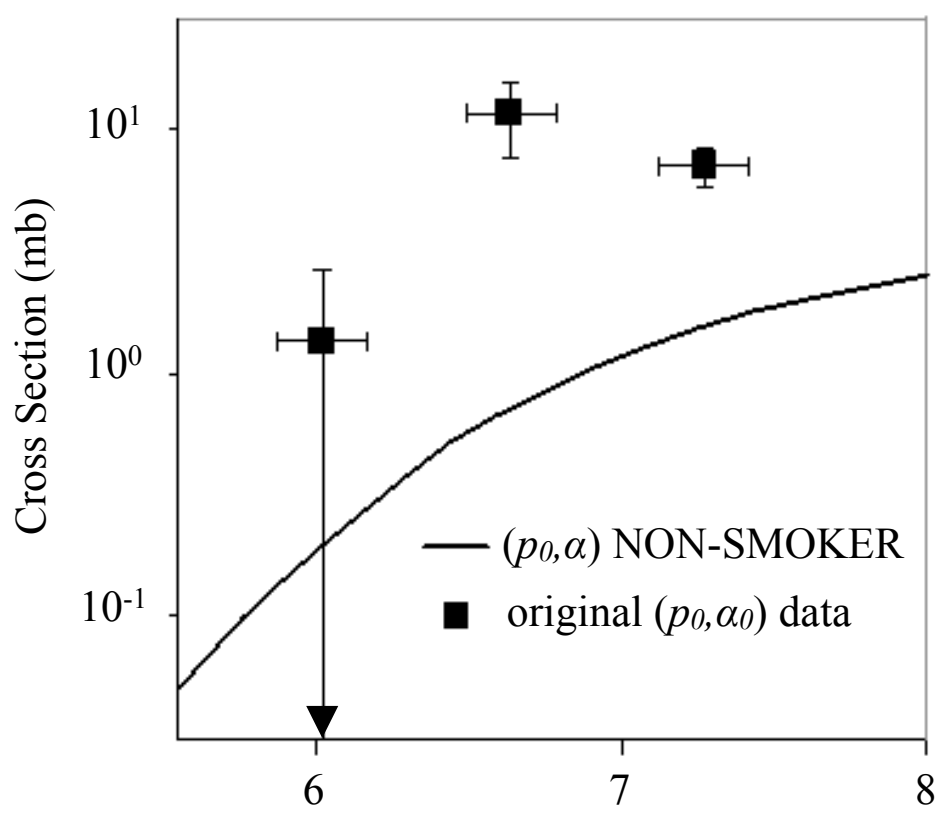

c. m. Energy (MeV)

Figure 2: Cross section as a function of c.m. energy for the ${ }^{33} \mathrm{Cl}\left(p_{0}, \alpha_{0}\right)^{30} \mathrm{~S}$ data (squares) and the NONSMOKER calculations $[14,15,16]$ for the ${ }^{33} \mathrm{Cl}\left(p_{0}, \alpha\right)^{30} \mathrm{~S}$ cross section (solid line). The experimental data only include ground state to ground state transitions, while the NON-SMOKER calculations include transitions to excited states. The vertical error bars indicate the uncertainties in the cross sections and the horizontal error bars indicate the energy spread of the beam in the target. Reproduced from [17].

the high-energy $\alpha$ branch is approximately 0.67 (e.g. for $p\left({ }^{33} \mathrm{Cl},{ }^{30} \mathrm{~S}\right) \alpha$ ) as determined via Monte Carlo simulations. The geometrical efficiencies of the DSSD and spectrograph were 0.82 and 0.38 , respectively, also determined via Monte Carlo simulations.

Normalization to the incident beam and target was done in several ways. For the $p\left({ }^{33} \mathrm{Cl},{ }^{30} \mathrm{~S}\right) \alpha$ measurement, the ratio of Rutherford scattering off the carbon component of the $\mathrm{CH}_{2}$ target at $2.5^{\circ}$, measured in the spectrograph, to the proton scattering at $8^{\circ}$, measured in the inner ring of the DSSD, was determined in a separate measurement during the experiment. The proton scattering was then monitored throughout the $(p, \alpha)$ experiment and using the ratio defined above with the calculated Rutherford cross section, normalization to the incident beam and target was achieved. In addition, for all the $(p, \alpha)$ measurements, the beam intensity and composition was checked every few hours by measuring it directly in the spectrograph after attenuation. Both methods of normalization agreed to within error. An additional Si surface barrier detector was used as an added check in both the $p\left({ }^{25} \mathrm{Al},{ }^{22} \mathrm{Mg}\right) \alpha$ and $p\left({ }^{37} \mathrm{~K},{ }^{34} \mathrm{Ar}\right) \alpha$ measurements to monitor the beam intensity and target thickness.

Once the $\alpha$-particle yield was corrected for efficiencies and normalized, the cross section could then be compared with theoretical calculations, such as those from the NON-SMOKER code $[14,15,16]$ (see e.g. Fig. 2 for the case of $\left.p\left({ }^{33} \mathrm{Cl},{ }^{30} \mathrm{~S}\right) \alpha[17]\right)$. 


\section{Conclusions}

The measured cross sections shown in Fig. 2 for the ${ }^{33} \mathrm{Cl}(p, \alpha)^{30} \mathrm{~S}$ reaction are up to a factor of four greater than the theoretical calculations produced using the NON-SMOKER code $[14,15,16]$. Furthermore, the cross sections measured in this experiment are only lower limits, as the measurement is only for the ground state to ground state transition, whereas NON-SMOKER also takes into account contributions from transitions to excited states in the final nucleus. By measuring the time-inverse reaction, it is not possible to determine these contributions for the $(\alpha, p)$ reaction of interest. The preliminary results for the cross sections of the $p\left({ }^{25} \mathrm{Al},{ }^{22} \mathrm{Mg}\right) \alpha, p\left({ }^{29} \mathrm{P},{ }^{26} \mathrm{Si}\right) \alpha$ and $p\left({ }^{37} \mathrm{~K},{ }^{34} \mathrm{Ar}\right) \alpha$ reactions also show deviations from NON-SMOKER calculations, although not always in the same direction. For example, the preliminary analysis of the $p\left({ }^{25} \mathrm{Al},{ }^{22} \mathrm{Mg}\right) \alpha$ reaction gives cross sections lower than NON-SMOKER; however, given that these cross sections are lower limits only, one cannot draw any definitive conclusion about how the total cross section, including contributions from excited states, would compare with NON-SMOKER calculations.

This disagreement is not altogether unexpected, as these theoretical rates are based on HauserFeshbach theory, which is dependent on the level densities of the compound nuclei. In this intermediate mass regime $(A=20-40)$, the level densities of even-even nuclei are low in the energy region of interest, and thus the reaction rate may be dominated by a few isolated resonances, making comparisons to Hauser-Feshbach based theories invalid. Indeed, the disagreement between these experimental results and theory underscores this point, and makes it clear that reaction rates based on experimental data are needed for stellar models that currently use these theoretical rates.

It should also be noted that the cross section measurements presented here are in an energy region that is above the astrophysically relevant region. Due to the lack of intense RIBs and the low cross sections that rapidly decrease with energy, it is impossible to measure these reactions in the astrophysically relevant energy regime with current techniques. However, if the discrepancies between measured and theoretically calculated cross sections persist at lower energies this may have significant effects on XRB nucleosynthesis. For example, if the cross section of ${ }^{30} \mathrm{~S}(\alpha, p){ }^{33} \mathrm{Cl}$ continues to be higher in the astrophysically relevant energy region, this would lead to a higher reaction rate and therefore a breakout of the ${ }^{30} \mathrm{~S}$ waiting-point, possibly eliminating the ${ }^{30} \mathrm{~S}(\alpha, p){ }^{33} \mathrm{Cl}$ reaction as an explanation for the double-peaked structure of some luminosity profiles. The effects on XRB nucleosynthesis that the new measured cross sections have will be published in a later work.

Given the importance of $(\alpha, p)$ reactions on $\alpha, p$-process waiting points in XRB nucleosynthesis additional work is clearly needed to advance the field. Measurements in the astrophysically relevant energy regime are desirable, as well as direct measurements of the forward $(\alpha, p)$ reactions. As facilities continue to produce more intense RIBs, these measurements will become feasible and direct studies of these reaction rates using the new HELIcal Orbit Spectrometer (HELIOS) at ATLAS [18] and the Array for Nuclear Astrophysical Studies with Exotic Nuclei (ANASEN) [19] are being explored. Measuring these and other key reaction rates, in addition to the other nuclear input that is needed for stellar modeling of XRBs, is crucial if we are to fully understand these astrophysical events. 


\section{Acknowledgments}

This work has been supported by JINA grant No. PHY0822648 and U.S. DOE contract DEAC02-06CH11357. We would also like to thank the ATLAS operations staff for making this study possible and Dr. Rauscher for helpful discussions regarding the NON-SMOKER code calculations.

\section{References}

[1] Schatz H and Rehm K E 2006 Nucl. Phys. A777 601

[2] Elomaa V V, Voobjev G K, Kankainen A, Batist L, Eliseev S, Eronen T, Hakala J, Jokinen A, Moore I D, Novikov Y N, Penttilä H, Popov A, Rahaman S, Rissanen J, Saastamoninen A, Schatz H, Seliverstov D M, Weber C and Äyastö J 2009 Phys. Rev. Lett. 102252501

[3] José J, Moreno F, Parikh A and Iliadis C 2010 Astrophys. J. Supp. Ser. 189204

[4] Fisker J L, Schatz H and Thielemann F K 2008 Astrophys. J. SS. 174261

[5] Sztajno M, van Paradijs J, Lewin W H G, Trümper J, Stollman G, Pietsch W and van der Klis M 1985 Astrophys. J. 299487

[6] Penninx W, van Paradijs J and Lewin W H G 1987 Astrophys. J. Lett. 32167

[7] Kuulkers E, Homan J, van der Klis M, Lewin W H G and Méndez M 2002 Astron. Astrophys. 382947

[8] Zhang G, Mendez M, Altamirano D, Belloni T M and Homan J 2009 Mon. Not. R. Astron. Soc. 389 368

[9] Bhattacharyya S and Strohmayer T E 2006 Astrophys. J. 641 L53

[10] Fisker J L, Thielemann F K and Wiescher M 2004 Astrophys. J. Lett. 60861

[11] Parikh A, José J, Moreno F and Iliadis C 2008 Astrophys. J. SS. 178110

[12] O'Brien S et al. 2009 AIP Conf. Proc. 1090288

[13] Paul M et al. 1989 Nucl. Inst. Meth. Phys. Res. A277 418

[14] Rauscher T and Thielemann F K 2000 Atomic Data and Nuclear Data Tables 751

[15] Rauscher T and Thielemann F K 2001 At. Data Nucl. Data Tables 7947

[16] Non-smoker code, http://nucastro.org/nonsmoker.html

[17] Deibel C M, Rehm K E, Clark J A, Figueira J M, Jiang C L, Kay B P, Lee H Y, Marley S T, Pardo R C, Patel N et al. 2009 ATLAS PAC proposal 1279

[18] Lighthall J C, Back B B, Baker S I, Freeman S J, Lee H Y, Kay B P, Marley S T, Rehm K E, Rohrer J E, Schiffer J P et al. 2010 Nucl. Instrum. Methods A622 97

[19] Matŏs M, Blackmon J C, Linhardt L E, Mondello L L, Johnson E, Rogachev G and Wiedenhoever I 2010 Proceedings of 11th Int. Symp. on Nuclei in the Cosmos (NIC-XI), Proceedings of Science 226 